\title{
REVISION OF THE GENUS LABISIA (MYRSINACEAE)
}

\author{
B. SUNARNO \\ Herbarium Bogoriense-Puslit Biologi, LIPI, Bogor, Indonesia
}

\begin{abstract}
SUMMARY
The genus Labisia (Myrsinaceae) is revised for the Flora Malesiana region. Seven species are recognised. Several earlier described species are synonymous to L. pumila and reduced to varieties of that species: var. alata, var. lanceolata, var. neriifolia and var. sessilifolia. A new record of L. longistyla is mentioned. Labisia malouiana and L. obtusifolia are excluded from the genus.
\end{abstract}

Key words: Labisia, Myrsinaceae, Malesia, new record, new varieties.

\section{INTRODUCTION}

The well-known Malesian genus Labisia Lindl. was described by Lindley (1845). Reinwardt (1826) published the genus Angiopetalum which antedates Lindley's description. A proposal to conserve the name of Labisia against Angiopetalum was published by Van Steenis (1948), which was later accepted by the Committee on Conservation of Names.

The habit of Labisia resembles that of Ardisia subg. Bladhia and it is not surprising that Labisia pumila was formerly referred to as Ardisia pumila (Blume, 1823, 1826; Miquel, 1856; Scheffer, 1867). In Mez's key to the genera of Myrsinaceae Labisia stands between the genera Tapeinosperma Hook.f. and Parathesis Hook.f. in the tribe Myrsineae because of the uniseriate ovules (Mez, 1902).

Typical for the genus are the numerous secondary veins \pm parallel to each other, the angle of divergence almost perpendicular. The small flowers with induplicate-valvate petals in which the stamens are wrapped undoubtedly characterise the genus as distinct from others in Myrsinaceae. The shape and size of mature corolla buds just before anthesis, petals, sepals, stamens and connective appendages, as well as the length of the styles, are here considered as specifically diagnostic. These characters were previously almost overlooked.

Mez (1902) recognised only a single species L. pumila informally indicating infraspecific taxa by using Greek letters $(\alpha, \beta, \mu)$ as did Scheffer (1867). Some botanists transcribed the symbols into formae (Airy Shaw, 1939, 1960), others into varieties (Valeton, 1907; Ridley, 1923b; Hochreutiner, 1925; Masamune, 1942; Fletcher, 1950; Stone, 1988), or retained the symbols (Pitard, 1930; Kanehira \& Hatusima, 1943).

New species were published several times (Linden \& Rodigas, 1885, 1892; Hallier, 1901, 1915; King \& Gamble, 1905; Ridley, 1908); however, except L. acuta and L. longistyla most of them are synonymous to L. pumila. Stone (1988) reviewed all 
the published taxa and also provided a synopsis of the species in Peninsular Malaysia. Very recently Sunarno (2002) published three new species from Sumatra.

The study of the specimens of Malesian Labisia available from BM, BO, K, KEP, L, OXFORD and UKMB showed that the genus also occurs in the Philippines, southern Thailand and Cambodia.

\section{LABISIA}

Labisia Lindl. (1845) 48, nom. cons.; Benth. \& Hook.f. (1876) 645; Clarke (1882) 518; Pax (1889) 93; Mez (1902) 172; King \& Gamble (1905) 115; Ridl. (1923a) 237; Pit. (1930) 801; Backer \&

Bakh.f. (1965) 200; B.C. Stone (1988) 43. - Type: Labisia pothoina Lindl. Angiopetalum Reinw. (1826) 7, nom. rej. - Type: Angiopetalum punctatum Reinw.

Small, erect, decumbent or creeping undershrubs. Vegetative and generative parts almost all covered with stellate-peltate trichomes, especially when young. Stems stout, terete or striate, very rarely branched. Leaves simple, alternately or spirally arranged, elliptic or ovate to obovate, upper surface glandular punctate, lower surface covered with peltate trichomes, midrib impressed above, secondary nerves numerous, widely spreading, almost parallel, rarely ascending, venation reticulodromus. Petioles (slightly or broadly) winged or not. Inflorescences axillary, panicles or racemes with corymb-like fascicles of 2-9 flowers. Flowers hermaphrodite, 5-merous, zygomorphic, glandular pusticulate, glabrous or thinly hairy. Sepals 5 , basally connate; lobes triangular, ovate or deltate, valvate or imbricate in bud. Petals 5, basally connate; lobes valvate in bud, ovate to narrowly ovate, induplicate. Stamens 5, epipetalous, enclosed by the petals; filaments short, flattened; anthers basifixed, opening longitudinally by slits, connective with short or long appendages, glandular pusticulate dorsally. Ovary superior; placenta discoid, ellipsoid or subglobose; ovules 6-8, uniseriate; style filiform, stigma small, notched. Fruits drupaceous, subglobose to globose, glandular punctate or punctatelineate, thinly to densely stellate when young. Seed 1, subglobose to globose, ribbed; endosperm smooth, embryo transverse, cylindric.

Distribution - A small genus with only seven species; throughout Malesia, also in Myanmar, Thailand and Cambodia.

Habitat \& Ecology - Lowland or montane forests, usually found in understorey, in secondary forest, bamboo forest and peat forest. Grows well in humus-rich soil, sandy soil, sandy loam soil, deep clayed soil, also in granite white sandy areas; from the beach to $2000 \mathrm{~m}$ altitude.

\section{KEY TO THE SPECIES OF LABISIA}

1a. Calyx lobes triangular or narrowly ovate, acuminate, valvate in bud ....... 2

b. Calyx lobes deltate, acute, acuminate, imbricate in bud . . . . . . . . . 7

2a. Flower buds just before anthesis subglobose or subovoid. Corolla lobes $2-3 \mathrm{~mm}$

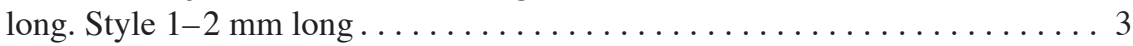

b. Flower buds just before anthesis ovoid. Corolla lobes 3-4 mm long. Styles 3-4

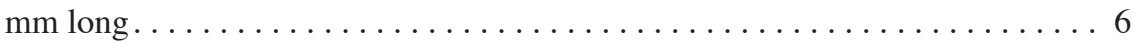

3a. Leaves many per plant, more than 5. Petiole winged. Corolla lobes $2-2.5 \mathrm{~mm}$ long. Anthers $0.8-1 \mathrm{~mm}$ long. Styles $1.5-2 \mathrm{~mm}$ long. . . . . . . . . . . 4 
b. Leaves few per plant, usually 3 or 4 . Petiole not winged. Corolla lobes c. $3 \mathrm{~mm}$ long. Anthers 1.5-1.7 mm long. Styles $1-1.5 \mathrm{~mm}$ long . . . . . 3. L. paucifolia

4a. Leaves chartaceous, apex acute, acuminate or obtuse. Clusters of flowers along the raceme short pedunculate. Styles $2-4 \mathrm{~mm}$ long. Fruits red when ripe. . . . . . 5

b. Leaves coriaceous, apex rounded. Clusters of flowers along the raceme long pedunculate. Styles $4-5 \mathrm{~mm}$ long. Fruits blue when ripe. . . . . . 2. L. longistyla

5a. Petiole not winged. Flower buds just before anthesis subovoid or ovoid. Corolla

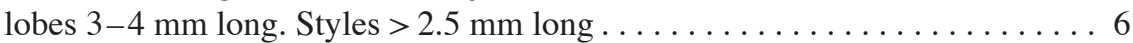

b. Petiole (broadly) winged or not. Flower buds just before anthesis subglobose. Corolla lobes $2-2.5 \mathrm{~mm}$ long. Style $<2.5 \mathrm{~mm}$ long . . . . . . . 5. L. pumila

6a. Flower buds just before anthesis subovoid. Corolla lobes c. $3 \mathrm{~mm}$ long. Connective appendage c. $0.3 \mathrm{~mm}$ long. Styles c. $3 \mathrm{~mm}$ long . . . . . . . 7. L. sumatrensis

b. Flower buds just before anthesis slightly conical or ovoid. Corolla lobes $3.5-4 \mathrm{~mm}$ long. Connective appendage $0.9-1 \mathrm{~mm}$ long. Styles $3.5-4 \mathrm{~mm}$ long . . . . . . . . $\ldots \ldots \ldots \ldots \ldots \ldots \ldots \ldots \ldots \ldots \ldots \ldots \ldots \ldots \ldots \ldots \ldots \ldots \ldots$ L. steenisiana

7a. Leaves narrowly elliptic, margin mostly entire, very rarely dentate to serrate. Flower buds just before anthesis conical. Anthers c. $2 \mathrm{~mm}$ long, connective appendage c. $0.5 \mathrm{~mm} \operatorname{long} \ldots \ldots \ldots \ldots \ldots \ldots \ldots \ldots \ldots \ldots \ldots$. L. acuta

b. Leaves broadly obovate or broadly oblong, margin dentate to serrate. Flower buds just before anthesis obclavate. Anthers c. $1 \mathrm{~mm}$ long, connective appendage c. 0.1 mm long . . . . . . . . posthumusiana

\section{Labisia acuta Ridl.}

Labisia acuta Ridl. (1908) 116; (1923a) 237; B.C. Stone (1988) 47. - Type: Ridley 13010 (holo K; iso SING n.v.), Sungau Tebrau, Johore.

Shrubs or undershrubs, erect or decumbent, up to $70 \mathrm{~cm}$ high. Stem slender, flexuous, striate. Leaves alternate; lamina (narrowly) elliptic, $10-15$ by $2.5-5 \mathrm{~cm}$, (sub)coriaceous, base acute, slightly decurrent, margin mostly entire, very rarely dentate or serrate, apex acute to obtuse, sometimes slightly acuminate, upper surface glabrous, greyish or brownish when dry, lower surface glabrous, green glaucous when dry, midrib narrowly impressed above, secondary nerves anastomosing at 1-3 mm from the margin; petioles $0.5-1 \mathrm{~cm}$ long, slightly winged. Inflorescences panicles or racemes, with corymb-like fascicles of 2-4 flowers, 4-6 cm long; peduncle 2-3 cm long; peduncle of the fascicles $0.5-1 \mathrm{~cm}$ long, sometimes longer or shorter. Corymb bracts deltate to triangular, very rarely ovate, leaf-like, 1-3 mm long. Floral bracts narrowly triangular, 1-2 mm long. Pedicel 1-2 mm long. Flowers white or pinkish white. Calyx lobes deltate, c. 1 $\mathrm{mm}$ long, cuspidate, imbricate in buds. Corolla lobes ovate, $4-5$ by c. $3 \mathrm{~mm}$, incurved margin c. $0.3 \mathrm{~mm}$ wide; in mature bud just before anthesis acutely conical, $3.5-4.5$ by $1.5-2 \mathrm{~mm}$. Stamens $2.5-2.7 \mathrm{~mm}$ long; filament c. $0.2 \mathrm{~mm}$ long; anthers c. $2 \mathrm{~mm}$ long, connective appendages c. $0.5 \mathrm{~mm}$ long. Ovary ovoid; placenta broadly ellipsoid; ovules 6 or 7, style filiform, 4-5 mm long. Fruit subglobose to globose, 7-9 mm diam. when fresh, or 4-5 mm when dry. Seed 3-4 mm diam., ribbed.

Distribution - Sumatra (Riau, Jambi, Central Sumatra, Bangka Isl.), Peninsular Malaysia, and Borneo (Sarawak, Central Kalimantan). 
Habitat \& Ecology - Swampy, white sand forest or peat forest. Altitude up to $400 \mathrm{~m}$. Flowering: September and November.

Note - The species is close to L. posthumusiana, but differs in the size and shape of leaves and in the flower parts (see note with L. posthumusiana). The venation is sometimes red at the lower surface. During a visit to Central Kalimantan, I found the plants growing near L. pumila on a river bank in a forest on granite soils.

\section{Labisia longistyla King \& Gamble}

Labisia longistyla King \& Gamble (1905) 117; Ridl. (1923a) 237; B.C. Stone (1988) 45. - Lectotype (here designated): Curtis 3750 (holo K; iso SING), Gap, Gn. Semangkok, 3000-4000 ft. alt., fl. May.

Small undershrubs, erect, older stems decumbent or creeping up to $50 \mathrm{~cm}$ long. Stems slender, rusty brown, creeping ones producing roots. Leaves usually 4 or 5 per plant, elliptic to suborbicular, $15-30$ by $5-10 \mathrm{~cm}$, coriaceous or subcoriaceous, base decurrent in a broad wing, margin entire, apex obtuse to round, sometimes slightly emarginated, upper surface glabrous, reddish brown or brown, lower surface glabrous, brown when dry, midrib narrowly impressed above, secondary nerves anastomosing at 1-3 $\mathrm{mm}$ from the margin; petioles broadly winged, wings $3-8 \mathrm{~cm}$ wide. Inflorescences panicles or racemes with few-flowered fascicles; peduncles $>7 \mathrm{~cm}$ long. Corymb and floral bracts small, subulate. Pedicels 4-5 mm long. Flowers pinkish or purplish. Calyx lobes ovate, acuminate, valvate in bud. Corolla lobes elliptic, $4-4.5$ by $2-3$ $\mathrm{mm}$, acuminate; buds (just before anthesis) subconical, 3-4 mm long. Stamen c. 1.5 $\mathrm{mm}$ long; filament c. $0.2 \mathrm{~mm}$ long; anthers ovate, sagittate, c. $1.5 \mathrm{~mm}$ long; connective appendage c. $0.3 \mathrm{~mm}$ long. Ovary globose; placenta ellipsoid or discoid, ovules 6 , uniseriate; style terete, slender, 3.5-4.5 mm long. Fruits globose, apex conical, blue when ripe, c. $8 \mathrm{~mm}$ diameter. Seed globose, conical at apex, coarsely ribbed, 4-5 $\mathrm{mm}$ diam.; endosperm transverse, curved.

Distribution - West Sumatra, Peninsular Malaysia.

Habitat \& Ecology - Montane peat forest (Peninsular Malaysia), hill forest (Sumatra). Altitude up to 1500 m. Flowering: March to June, August to October, December; fruiting: August, October.

Note - Older creeping stems are often deeply buried in high land peat, sending young shoots up to the peat surface. Okada \& Mori 1214 is the first record for Sumatra.

\section{Labisia paucifolia King \& Gamble}

Labisia paucifolia King \& Gamble (1905) 117; Ridl. (1923a) 237; B.C. Stone (1981) 122; (1988) 47. - Lectotype (here designated): King's collector 8078 (n.v.).

Small undershrubs, up to $45 \mathrm{~cm}$ high. Stems slender, striate, older part creeping, shoot erect. Leaves few, usually 4 or 5 per plant, ovate to suborbiculate, 3-5 by 4-6 cm, base obtuse to round, slightly decurrent, margin entire to slightly crenate, apex acute to acuminate, upper surface glabrous, grey or brown, lower surface glabrous, grey when dry, midrib narrowly impressed above, secondary nerves anastomosing at $1-3 \mathrm{~mm}$ from the margin; petioles 3-8 cm long, terete, base thickened. Inflorescences racemes or panicles with few-flowered fascicles, usually $<5 \mathrm{~cm}$ long. Corymb and floral bracts 
subulate. Pedicels 2-3 mm long. Flowers pinkish white. Calyx lobes ovate, acuminate, valvate in bud. Corolla lobes elliptic, $3-3.2$ by c. $1.5 \mathrm{~mm}$, top acuminate; buds (just before anthesis) subovoid, c. $3 \mathrm{~mm}$ long. Stamens c. $2.2 \mathrm{~mm}$ long; filaments c. $0.2 \mathrm{~mm}$ long; anthers c. $1.5 \mathrm{~mm}$ long; connective appendage c. $0.5 \mathrm{~mm}$ long. Ovary globose; placenta ellipsoid or discoid; ovules 7 or 8 , uniseriate; style subulate $1-1.5 \mathrm{~mm}$ long. Fruits globose, sometimes conical at apex, scarlet when ripe, c. $8 \mathrm{~mm}$ diameter. Seeds globose, conical at apex, coarsely ribbed, 3-4 mm diameter.

Distribution - North Sumatra (Asahan), Peninsular Malaysia.

Habitat \& Ecology - Montane shady forest with humus-rich soil. Altitude c. $1500 \mathrm{~m}$.

Note - Labisia paucifolia is closer to L. sumatrensis than to L. steenisiana. Clear differences are observed in the morphological structure of the flowers as the shape of the corolla in the mature buds, the size of the petals, styles and stamens. Satisfactory identification of sterile or fruiting specimens of these three species in the herbarium is hardly possible.

\section{Labisia posthumusiana Sunarno}

Labisia posthumusiana Sunarno (2002) 121, fig. - Type: Posthumus 777 (holo BO; iso L), Sumatra, Prov. Jambi, Selemoekoe.

Erect or decumbent undershrubs, $60 \mathrm{~cm}$ high. Stems rather stout. Leaves many, broadly elliptic or broadly obovate, $16-22$ by $6-12 \mathrm{~cm}$, very rarely smaller, subcoriaceous, base acuminate, decurrent, margin dentate, apex acute to obtusely acuminate or rounded, emarginate, upper surface glabrous, brownish and dull when dry, lower surface glabrous, brownish, slightly glaucous, midrib narrowly impressed above, secondary nerves anastomosing at 5-7 $\mathrm{mm}$ from the margin; petiole $0.5-1.5 \mathrm{~cm}$ long, slightly winged. Inflorescences axillary, panicles or racemes with corymb-like fascicles with 2-5 flowers, 10-15 cm long; peduncle 4-5 cm long; corymb peduncle 4-5 mm long. Corymb and floral bracts nearly equal, triangular, c. $1 \mathrm{~mm}$ long. Flower white or pinkish white. Pedicels c. $2 \mathrm{~mm}$ long. Sepals 5, connated only at base; segments valvate; lobes imbricate in mature flower buds, triangular, slightly cuspidate at apex, c. $1 \mathrm{~mm}$ long. Petals 5 , obclavate in mature buds, $4-4.5 \mathrm{~mm}$ long; lobes (narrowly) ovate, $4.5-5$ by $1-1.5$ mm. Stamens 5, c. $1.2 \mathrm{~mm}$ long, filaments c. $0.1 \mathrm{~mm}$ long; anthers elliptic, c. $1 \mathrm{~mm}$ long, connective appendage c. $0.1 \mathrm{~mm}$ long. Ovary ovoid; placenta broadly ellipsoid, ovules 6; style filiform, 4-5 mm long. Fruits drupaceous, young fruit subglobose.

Distribution - Sumatra (Jambi, Pasirmayang, Bukit Cengkeembun, Indragiri Highlands).

Habitat \& Ecology — Primary forest and swampy forest. Altitude 50-300 m, probably higher, up to 700 m. Flowering: August, December; fruiting: February, October, December.

Note - Leaves dark green, venation red. Peduncle red. Fruits light green.

\section{Labisia pumila (Blume) Fern.-Vill.}

Labisia pumila (Blume) Fern.-Vill. (1880) 123; Merr. (1907) 296; (1923) 266; Airy Shaw (1961) 206; Backer \& Bakh.f. (1965) 200; B.C. Stone (1988) 47. - Labisia pumila (Blume) Benth. \& Hook.f. ex Jacks. (1895) 17; Mez (1902) 171; Pit. (1930) 802; Steenis (1948) 109. — Labisia pumila (Blume) Mez ex Airy Shaw (1939) 516. - Ardisia pumila Blume (1823) 44; (1826) 688; Miq. (1856) 1028. - Lectotype (here designated): Blume s.n. (holo L, HLB 900.211-151), Java. 
Angiopetalum punctatum Reinw. (1826) 7. - Allopetalum punctatum Reinw. ex Scheff. (1867) 92. - Ardisia bantamica Zipp. ex Scheff. (1867) 92. - Labisia punctata (Reinw.) Airy Shaw (1960) 320. - Lectotype (here designated): Zippelius s.n. (holo L, HLB 308.133-614), Java.

Ardisia spicata Wall. ex DC. (1834) 135. - Type: Wallich 2773 (holo n.v.), Singapore.

Labisia pothoina Lindl. (1845) 48; Ridl. (1923a) 237. - Lectotype (here designated): Griffith 3559 (holo K), Malacca, Herb. Hooker, 1867.

Ardisia pumila Blume $\beta$ lanceolata Scheff. (1867) 92. - Labisia pumila (Blume) Fern.-Vill. var. lanceolata (Scheff.) Mez (1902) 172. - Labisia pothoina Lindl. var. lanceolata (Scheff.) King \& Gamble (1905) 116; Ridl. (1923a) 237. - Labisia pumila (Blume) Fern.-Vill. forma lanceolata (Scheff.) Mez ex Airy Shaw (1939) 516; (1960) 416. - Labisia punctata (Reinw.) Airy Shaw forma lanceolata (Scheff.) Airy Shaw (1960) 320. - Lectotype (here designated): Korthals s.n. (holo L, L 900.211-155), Borneo, Martapoera, fl.

Ardisia pumila Blume $\gamma$ alata Scheff. (1867) 93. - Labisia alata (Scheff.) N.E. Br. (1886) 123, t. 605. - Labisia pumila (Blume) Fern.-Vill. var. alata (Scheff.) Mez (1902) 172; H.R. Fletcher (1950) 117. - Labisia pothoina Lindl. var. alata (Scheff.) King \& Gamble (1905) 116; Ridl. (1923a) 237. - Lectotype (here designated): Reinwardt s.n. (holo L, L 900.211-155), Java.

Labisia serrulata Hallier f. ((1887) 257 (nom. nud.)); (1901) 667 (descr.); B.C. Stone (1988) 51. - Lectotype (here designated): Jaheri s.n. (sterile plant, Sumatra, Deli, Gunung Tandjung. Living plant cultivated in Bogor Bot. Gard., no longer extant, Bull. Boiss. Ser. 2. 1.7 (1901) 667, t. 10 and description.

Labisia smaragdina L. Linden \& Rodigas (1892) 91, t. 160. - Lectotype (here designated): Anon. s.n. (Borneo. Plant cultivated in Int. Hort. Gard. Brussel, Belgium, no longer extant, Ill. Hort. 39 (1892) 140, t. 109, figure and description.

Labisia neriifolia Hallier f. (1901) 669. - Neotype (here designated): Hallier 492 (holo BO), Gn. Damoes, Jan. 1894.

Labisia pumila (Blume) Fern.-Vill. var. lanceolata (Scheff.) Mez forma sessilifolia Valeton (1907) 43; B.C. Stone (1988) 50. - Labisia sessilifolia (Valeton) Hallier f. (1915) 6. - Type: Koch s.n. (holo L, HLB 907.101-23; iso BO), West New Guinea.

Labisia ovalifolia Ridl. (1923b) 76; B.C. Stone (1988) 51. - Type: Moh. Nur 7395 (holo SING, photo seen), Sumatra, Gn. Sebayak (Sibayak), fl. Aug. 1921.

Labisia pumila (Reinw.) Airy Shaw forma gladiata Airy Shaw (1939) 517. - Labisia pumila (Reinw.) Airy Shaw var. gladiata (Airy Shaw) Masam. (1942) 585. - Labisia punctata (Reinw.) Airy Shaw forma gladiata (Airy Shaw) Airy Shaw (1960) 320. - Type: Synge 50 (holo K), Sarawak, 4th Div., Marudi, near sea level, fl. July.

Undershrubs, erect $10-40 \mathrm{~cm}$ high or prostrate up to $60 \mathrm{~cm}$ long or longer. Leaves more than 5 per plant; lamina (narrowly or broadly) elliptic or ovate $10-30$ by $1.3-11 \mathrm{~cm}$, herbaceous, chartaceous to subcoriaceous, base acute or obtuse, more or less decurrent, or sometimes slightly cordate, margin serrate, dentate, crenulate, subentire or entire, apex acuminate, acute or obtuse, midrib narrowly impressed above, secondary nerves anastomosing at 1-4 $\mathrm{mm}$ from margin; petioles either long, terete, slightly to broadly winged. Inflorescences axillary, panicles or racemes, with corymb-like fascicles of 2-9 flowers, 5-25 cm long. Corymb and floral bracts narrowly triangular, 1-3(-5) mm long. Pedicels 1-3 mm long. Flowers white or pinkish white. Calyx connate for $1 / 3$ to $1 / 2$ of its length; segments valvate; lobes triangular $0.5-1 \mathrm{~mm}$ long, acute to acuminate. Corolla connate near the base; segments valvate; lobes ovate, $2-2.5$ by 1-1.5 mm; apex subacute; in mature buds, just before anthesis subglobose, $1.5-2 \mathrm{~mm}$ long, top obtuse. Stamens 1-1.5 mm long; anthers sagittate, $0.8-1 \mathrm{~mm}$ long; filament c. $0.2 \mathrm{~mm}$ long; connective appendages $0.1-0.3 \mathrm{~mm}$ long, backside glandular pusticulate. Ovary subglobose, ovoid, broadly ellipsoid or depressedly deltoid; placenta subglobose, (broadly) ellipsoid or discoid; ovules 6 or 7; style subulate, 1.5-2 mm long. 
Fruits subglobose, 4-5 mm diam., ribbed with linear or elongate glandular streaks, green when young, red when ripe. Seed 1, subglobose, 3-4 mm diam., ribbed.

Distribution - Sumatra, Java, Kalimantan, Sulawesi, Moluccas, Lesser Sunda Islands (Flores), West New Guinea (Irian Jaya).

Habitat \& Ecology - Mostly in shaded areas on humus-rich soils, sandy loam, sometimes in deep clay soil or granite soils. Flowering and fruiting throughout the year.

Notes -1 . The combinations L. pumila (Blume) Benth. \& Hook.f. as indicated by Index Kewensis (1894), and L. pumila (Blume) Mez ex Airy Shaw (1939) are obviously superfluous to L. pumila (Blume) Fern.-Vill. (1880).

2. Labisia smaragdina L. Linden \& Rodigas, was described on the basis of a living plant cultivated in the International Horticultural Garden of Brussel, Belgium. In the present collection of the garden the plant is no longer extant and no herbarium specimen of this collection is available. Therefore, the description and the figure of the species in the protologue is here designated as the lectotype.

3. Labisia serrulata Hallier f. was described on the basis of a living plant, Jaheri s.n., cultivated in Bogor Botanic Garden in 1895. Since no specimen of Jaheri at BO is similar to the description of the taxon, the figure and description of the species by Hallier is here designated as the lectotype. Stone (1988) considered L. ovalifolia as a synonym of $L$. serrulata which he took for a distinct species. I suggest that both species are synonymous to L. pumila.

4. The type of L. pothoina was a living plant cultivated at the Royal Botanical Gardens, Kew, but no specimen of Lindley's plant could be found at Kew. Griffith 3559 was also cited by Lindley (1845) and here designated as the lectotype.

5. Labisia neriifolia Hallier f. was described on the basis of a living plant cultivated at Bogor Botanic Garden from West Borneo. The plant is no longer extant and no preserved dry specimen is available at BO. Specimen Hallier 492 from Gn. Damus, a place not far from the type locality and collected in the same year, conforms to the description and figure of the species, and is here designated as the neotype.

6. Labisia sessilifolia is confirmed to be a synonym of L. pumila; however, the pulverulentness of young fruits is uncommon.

7. Eight varieties are recognised, seven in Indonesia and one, var. gladiata, from North Borneo (Sarawak).

\section{KEY TO THE VARIETIES}

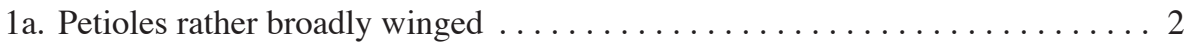

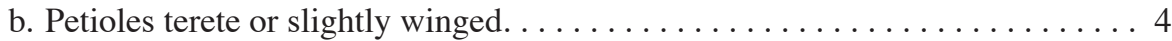

2a. Petioles 1-9 mm long, wings 8-12 mm wide. Anthers 1-1.2 mm long. Ovary depressedly deltoid or depressedly ellipsoid, placenta discoid . . . . . . 3

b. Petioles $5-12 \mathrm{~cm}$ long, wings $3-5 \mathrm{~mm}$ wide. Anthers c. $0.8 \mathrm{~mm}$ long. Ovary broadly

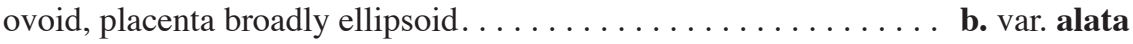

3a. Petioles up to $1 \mathrm{~cm}$ long, wings $7-9 \mathrm{~mm}$ wide. Anthers c. $1 \mathrm{~mm}$ long. Ovary depressedly discoid . . . . . . . . . . . . discoplacenta

b. Petioles 8-9 cm long, wings 19-12 mm wide. Anthers c. $1.2 \mathrm{~mm}$ long. Ovary depressedly ellipsoid $\ldots \ldots \ldots \ldots \ldots \ldots \ldots$ f. var. malintangensis 


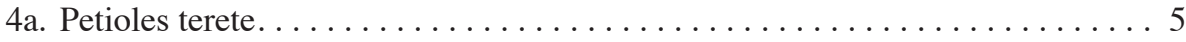

b. Petioles slightly winged. ....................... 6

5a. Leaf index 2-3. Anthers c. $0.8 \mathrm{~mm}$ long. Ovary ovoid, placenta ellipsoid.

e. var. lanceolata

b. Leaf index 5-9. Anthers c. $1 \mathrm{~mm}$ long. Ovary and placenta subglobose . . . . . . . g. var. neriifolia

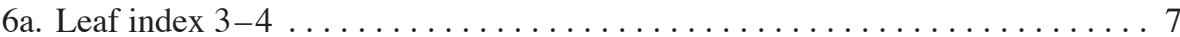

b. Leaf index $5-6 \ldots \ldots \ldots \ldots \ldots \ldots \ldots \ldots \ldots \ldots \ldots \ldots$ d. var. gladiata

7a. Petal lobes c. $2.5 \mathrm{~mm}$ long. Anthers c. $1.2 \mathrm{~mm}$ long. Ovary subglobose . . . . . .

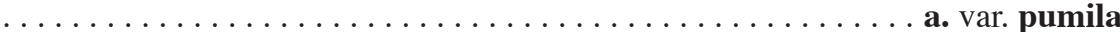

b. Petal lobes c. $2 \mathrm{~mm}$ long. Anthers c. $0.8 \mathrm{~mm}$ long. Ovary ovoid, placenta ellipsoid. . . . . . . . . . . . . . . . . . . . . . . . . . h. var. sessilifolia

\section{a. var. pumila}

Labisia pumila (Blume) Fern.-Vill. var. pumila. - Labisia pumila (Blume) Fern.-Vill. a genuiana Mez (1902) 172. - Labisia pumila (Blume) Fern.-Vill. forma genuiana Mez ex Airy Shaw (1939) 516. - Labisia punctata (Reinw.) Lindl. forma pumila (Blume) Airy Shaw (1960) 320. - Ardisia pumila Blume a genuiana Scheff. (1867) 92. - Lectotype (here designated): Blume s.n. (lecto L, HLB 900.211-151), Java.

Petioles slightly winged, 4-15 cm long. Anthers c. $1.2 \mathrm{~mm}$ long. Ovary subglobose, placenta subglobose.

Distribution - Thailand; Malesia: Sumatra, Peninsular Malaysia, Java, Borneo, Philippines, Moluccas.

Habitat \& Ecology - Shady rain forests or the edge of swampy forests on humusrich or acid sandy soils, at 20-1500 m altitude. Flowering throughout the year, except March and July.

b. var. alata (Scheff.) Mez

Labisia pumila (Blume) Fern.-Vill. var. alata (Scheff.) Mez (1902) 172; H.R. Fletcher (1950) 117. - Ardisia pumila Blume $\gamma$ alata Scheff. (1867) 92. - Labisia alata (Scheff.) N.E. Br. (1886) 123, t. 605. - Labisia pothoina Lindl. var. alata (Scheff.) King \& Gamble (1905) 116; Ridl.(1923a) 237. - Lectotype (here designated): Reinwardt s.n. (holo L, L 900.211-155), Java.

Angiopetalum punctatum Reinw. (1826) 7. - Allopetalum punctatum Reinw. ex Scheff. (1867) 93. - Labisia punctata Reinw. forma punctata Airy Shaw (1960) 320.

Labisia ovalifolia Ridl. (1923b) 76; B.C. Stone (1988) 51. - Type: Moh. Nur 7395 (holo SING, seen photo), Sumatra, Gn. Sebayak (Sibayak), fl. Aug. 1921.

Labisia serrulata Hallier f. ((1887) 257, nom. nud.); (1901) 667 (descr.); B.C. Stone (1988) 51. - Lectotype (here designated): Jaheri s.n. (lecto fig. and descr.), Sumatra, Tandjung Gunung, Deli, living plant cultivated in Bogor Bot. Gard., no longer extant, Bull. Boiss. Ser. 2, 1, 7 (1901) 667 , t. 10, description.

Petioles 5-12 cm long, wing 3-5 mm wide at each side. Anthers c. $0.8 \mathrm{~mm}$ long. Ovary broadly ovoid, placenta broadly ellipsoid.

Distribution - Myanmar, Thailand; Malesia: Sumatra, Peninsular Malaysia, Java, Borneo, Philippines, Lesser Sunda Islands.

Habitat \& Ecology - Lowland primary forests at shady places or in secondary forests on humus-rich soils. 
Note - Var. alata is close to var. malintangensis and var. discoplacenta, but differs in the length of anthers, the shape of ovary and placenta, and also the size of the petiole and its wings.

\section{c. var. discoplacenta Sunarno, var. nov. - Fig. 1}

Var. alata affinis sed petioles breviora, antheris et ovaries et placentas atque distincta. - Typus: Afriastini 1288 (holo BO), West Kalimantan, Nanga Semangit, Selimbau District, Kapuas Hulu.

Petioles c. $1 \mathrm{~cm}$ long, rather broadly winged. Anthers c. $1 \mathrm{~mm}$ long. Ovary \pm conical, placenta discoid.

Distribution - Known from the type specimen only.

Habitat \& Ecology - Primary forest.

Note - Var. discoplacenta is close to var. alata, which also has short petioles, but differs in the size of the anthers and in the shape of ovary and placenta.

d. var. gladiata (Airy Shaw) Masam.

Labisia pumila (Blume) Fern.-Vill. var. gladiata (Airy Shaw) Masam. (1942) 585. - Labisia pumila (Blume) Fern.-Vill. forma gladiata Airy Shaw (1939) 517. - Labisia punctata (Reinw.) Airy Shaw forma gladiata (Airy Shaw) Airy Shaw (1960) 320. - Type: Synge 50 (holo K), Sarawak, Marudi, 4th Div., near sea level, fl. July.

Petioles slightly winged, 2-4 cm long. Anthers c. $1 \mathrm{~mm}$ long. Ovary ovoid, placenta ellipsoid.

Distribution - Sarawak.

\section{e. var. lanceolata (Scheff.) Mez}

Labisia pumila (Blume) Fern.-Vill. var. lanceolata (Scheff.) Mez (1902) 172; B.C. Stone (1988) 45. - Ardisia pumila Blume $\beta$ lanceolata Scheff. (1867) 92. - Labisia pumila (Blume) Fern.-Vill. forma lanceolata (Scheff.) Mez ex Airy Shaw (1939) 516. - Labisia pothoina Lindl. var. lanceolata (Scheff.) King \& Gamble (1905) 116; Ridl. (1923a) 237. - Labisia punctata (Reinw.) Airy Shaw forma lanceolata (Scheff.) Airy Shaw (1960) 320. - Lectotype (here designated): Korthals s.n. (holo L, HLB 900.211-158), Borneo, Martapoera, fl.

Petioles terete, 6-21 cm long. Anthers c. $0.8 \mathrm{~mm}$ long. Ovary ovoid, placenta ellipsoid.

Distribution - Sumatra, Peninsular Malaysia, Java, Borneo, Sulawesi, Moluccas, Lesser Sunda Islands (Flores).

Habitat \& Ecology - Common in shady primairy forests, secondary and mossy forests. Growing well on humus-rich soils, granite and acid soils at 50-1200 m altitude.

\section{f. var. malintangensis Sunarno, var. nov. - Fig. 2}

Var. alata affinis sed antheris longiora, ovaris depresso-ellipsoidis, placentas discoidea differt. - Typus: Bünnemeijer 3632 (holo BO; iso K, L), Sumatra west coast, Mt Malintang, northwest of Helling, $1100 \mathrm{~m}$ alt., fl. July.

Petioles 8-9 cm long, wings 8-12 mm wide. Anthers c. $1.2 \mathrm{~mm}$ long. Ovary transversely ellipsoid, placenta discoid. 


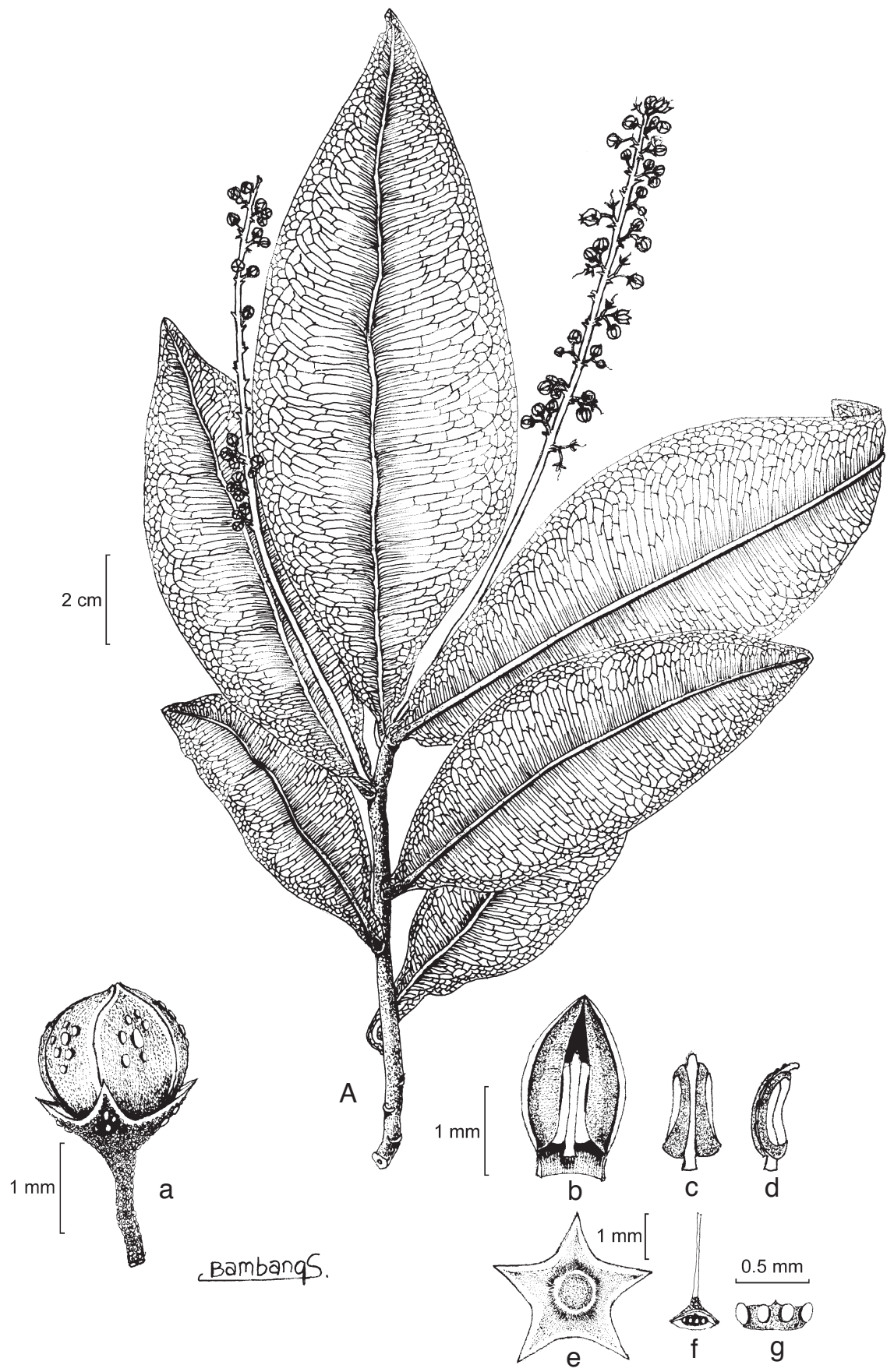

Fig. 1. Labisia pumila (Blume) Fern.-Vill. var. discoplacenta Sunarno. A. Habit; a. flower bud; b. front side view of detached petal with enclosed stamen; c. stamen, frontal view; d. stamen, side view; e. calyx, inside view; f. opened ovary; g. placenta and ovules (all: Afriastini 1288). 


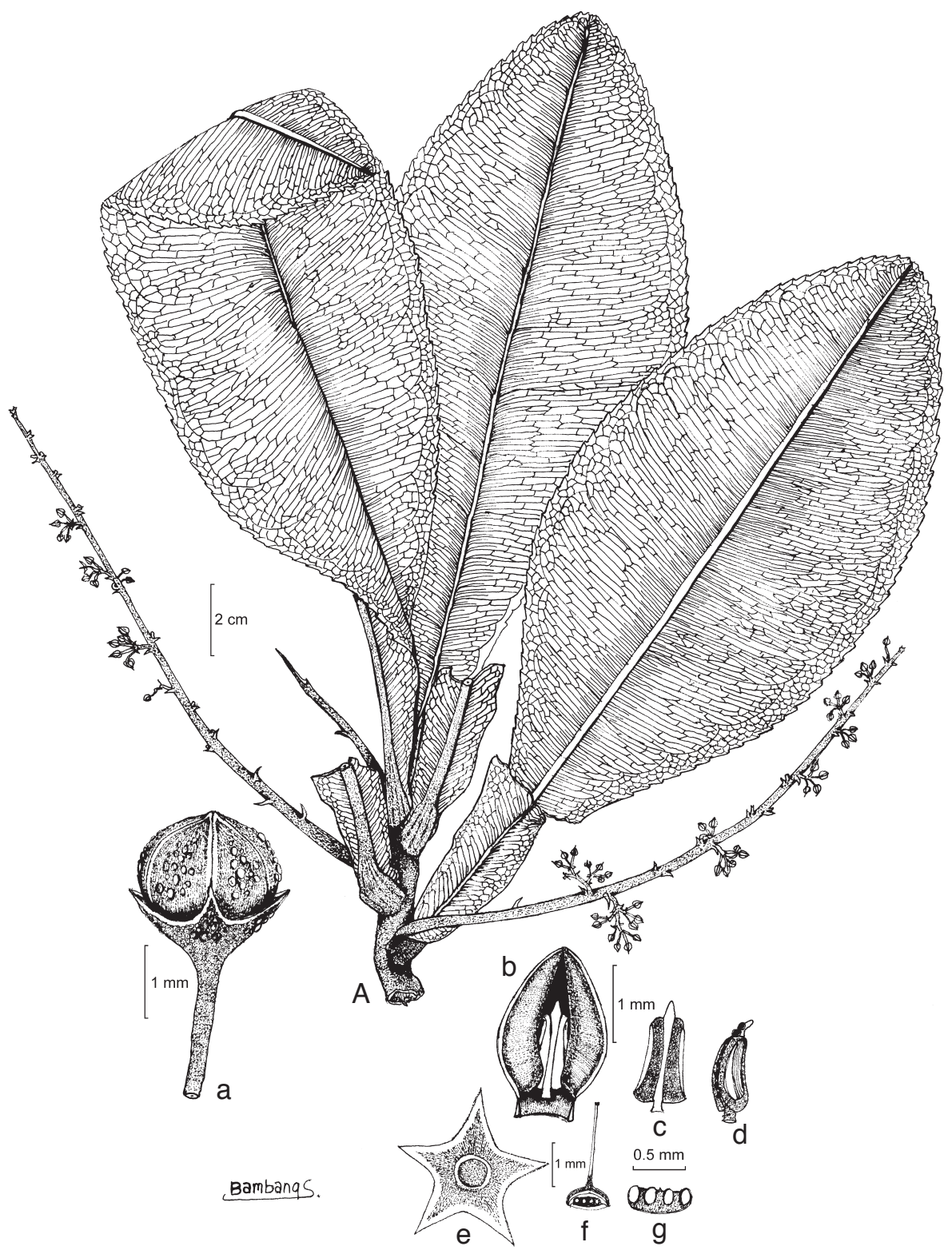

Fig. 2. Labisia pumila (Blume) Fern.-Vill. var. malintangensis Sunarno. A. Habit; a. flower bud; b. front side view of detached petal with enclosed stamen; c. stamen, frontal view; d. stamen, side view; e. calyx, inside view; f. opened ovary; g. placenta and ovules (all: Bünnemeijer 3632). 
Distribution - Sumatra, Peninsular Malaysia.

Habitat \& Ecology - In open places inside primary forests.

Note - Superficially var. malintangensis resembles var. alata, but differs in the broader wings of the petioles and the shape and size of the flower parts. The ovary and placenta are similar to that of var. discoplacenta which can easily be distinguished by the larger petal lobes, longer anthers and connective appendage. The epithet refers to the type locality.

g. var. neriifolia (Hallier f.) Sunarno, comb. \& stat. nov. - Fig. 3

Basionym: Labisia neriifolia Hallier f. (1901) 669. - Neotype (here designated): Hallier 492 (holo BO), G. Damoes, fr., Jan. 1894.

Petioles terete, 5-12 cm long. Anthers c. $1 \mathrm{~mm}$ long. Ovary and placenta subglobose.

Distribution - Borneo, Sulawesi, Moluccas.

Habitat \& Ecology — Primary and secondary forests on sandy loamy soils. Flowering: July to November.

Note - Var. neriifolia is apparently close to var. gladiata from Sarawak; the latter variety, however, has a winged petiole.

h. var. sessilifolia (Valeton) Sunarno, comb. \& stat. nov. - Fig. 4

Basionym: Labisia pumila (Blume) Fern.-Vill. var. lanceolata (Scheff.) Mez forma sessilifolia Valeton (1907) 43. - Labisia sessilifolia (Valeton) Hallier f. (1915) 6. - Type: Koch s.n. (holo L; iso BO), Merauke, southwest New Guinea, fl. 1904/1905.

Labisia pumila (Blume) Fern.-Vill. $\beta$ alata auct. non Mez: Kanehira \& Hatusima (1943) 225.

Petioles 2-4 cm long, wings 1-2 mm wide. Anthers c. $0.8 \mathrm{~mm}$ long. Ovary subglobose, placenta ellipsoid.

Distribution - Moluccas, New Guinea (Papua Barat, probably also in Papua New Guinea).

Habitat \& Ecology - Shady rain forests or the edge of swampy forests; 75-300 m altitude. Flowering: March, July, November.

Note - Var. sessilifolia is close to var. pumila, but differs in the lateral nerves and in the shape and size of flower structures.

\section{Labisia steenisiana Sunarno}

Labisia steenisiana Sunarno (2002) 123, f. 2 - Type: Van Steenis 6310 (holo BO; iso L), Sumatra, Kab. Aceh Tenggara, Gayo land, Mt Kemiri east slope, Camp 1 to 2, 1800 m alt., fl. March, 1937.

Undershrubs, erect to $20-25 \mathrm{~cm}$ high or decumbent up to $50 \mathrm{~cm}$ long. Stem slender. Leaves spirally arranged or apparently alternate; lamina ovate, $5-12$ by $2-6 \mathrm{~cm}$, subcoriaceous, base acute to obtuse, decurrent or slightly so, margin entire to crenulate; apex acute to acuminate, midrib narrowly impressed above, strongly prominent beneath, secondary nerves anastomosing at $4-5 \mathrm{~mm}$ from margin; petioles $3-5 \mathrm{~cm}$ long, terete or slightly winged (marginate), base thickened. Inflorescences axillary, panicles, 6-20 cm long. Bracts and bracteoles narrowly triangular, 2-3 $\mathrm{mm}$ long; peduncles $3-9 \mathrm{~cm}$ long. Flowers white or pinkish white; pedicels $2-3 \mathrm{~mm}$ long. Calyx lobes 


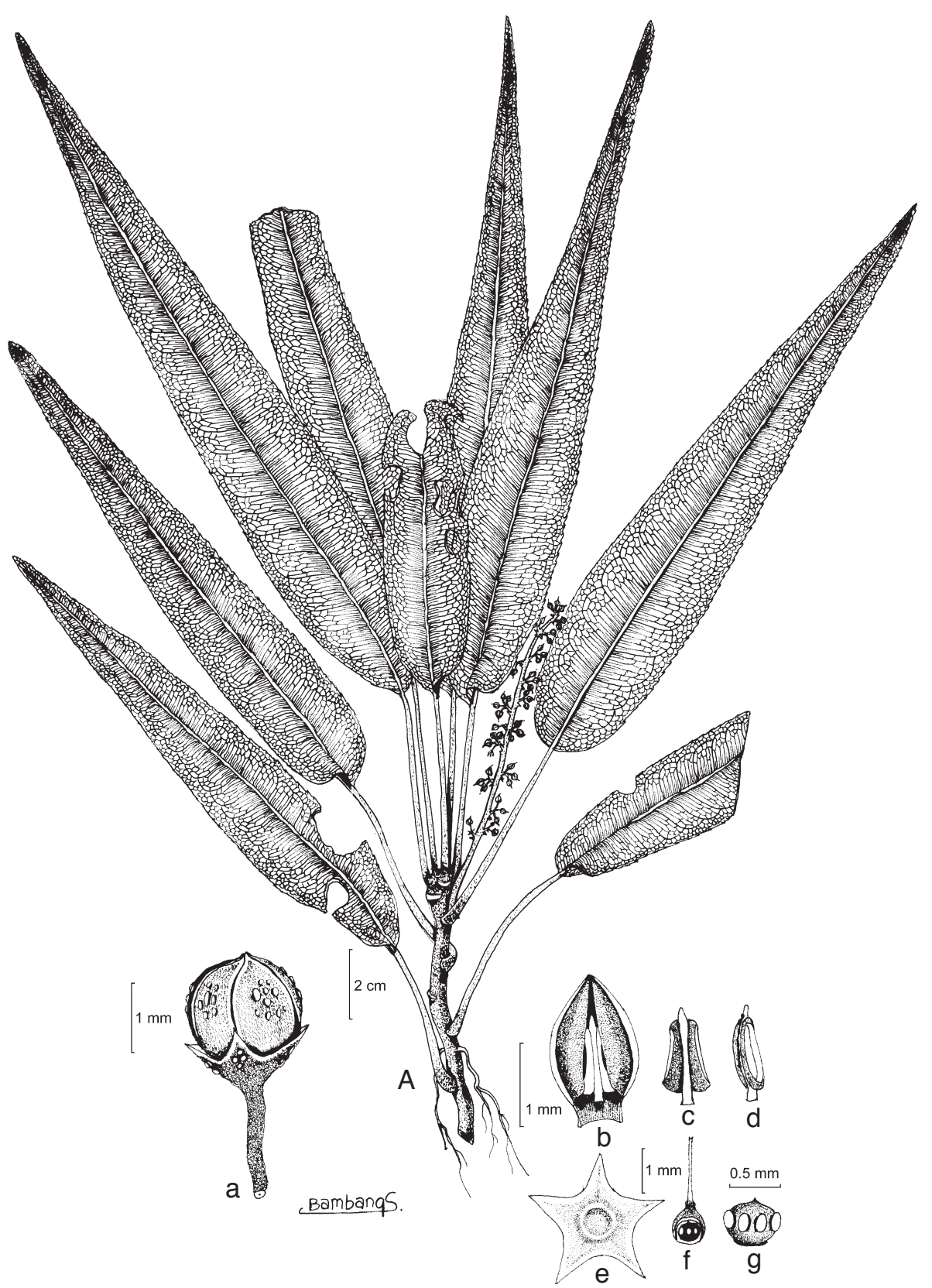

Fig. 3. Labisia pumila (Blume) Fern.-Vill. var. neriifolia (Hallier f.) Sunarno. A. Habit; a. flower bud; b. front side view of a detached petal with enclosed stamen; c. stamen, backside view; d. stamen, side view; e. calyx, inside view; f. opened ovary; g. placenta and ovules (all: Rachmat 471). 


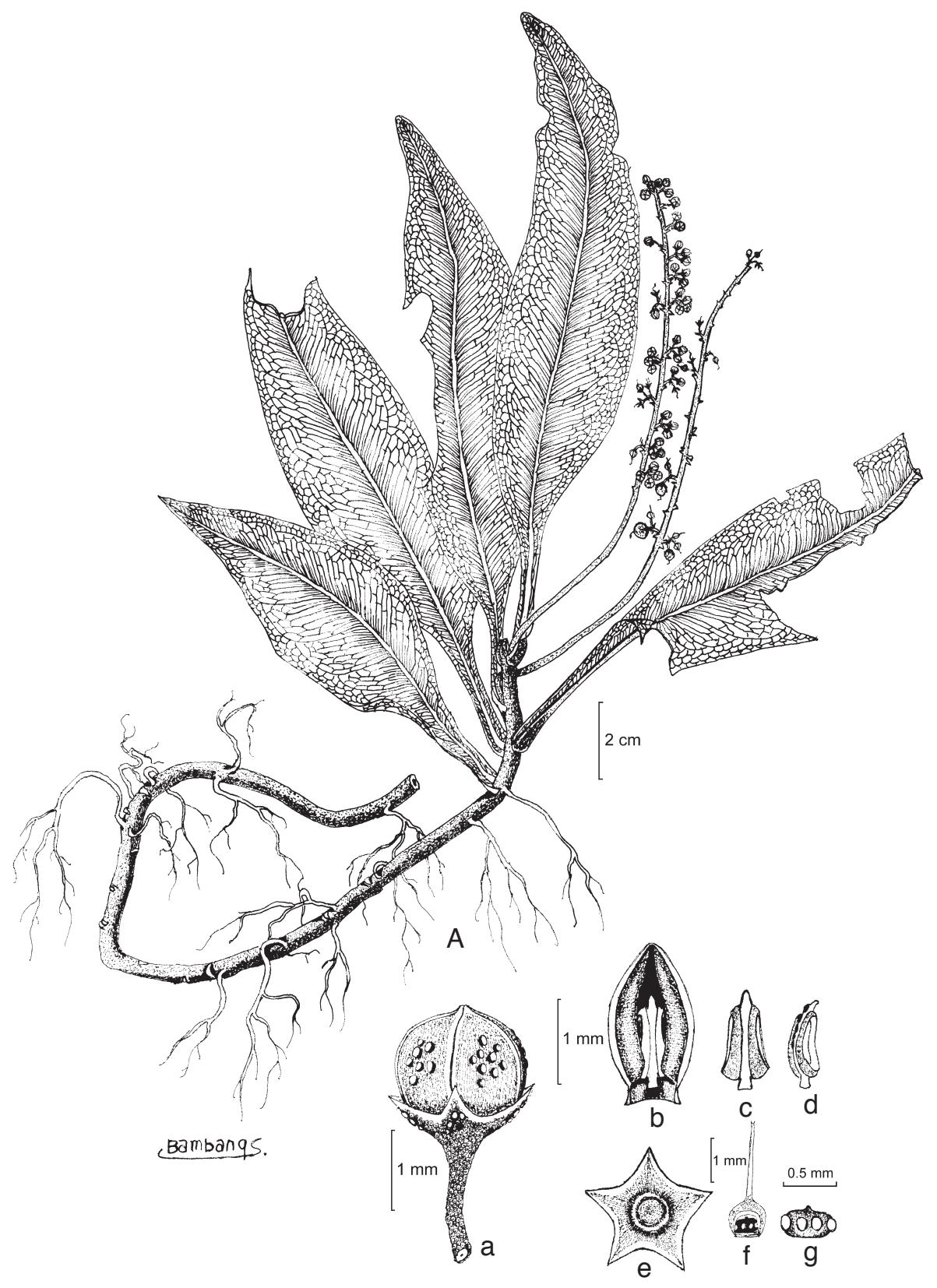

Fig. 4. Labisia pumila (Blume) Fern.-Vill. var. sessilifolia (Valeton) Sunarno. A. Habit; a. flower bud; b. front side view of a detached petal with enclosed stamen; c. stamen, backside view; d. stamen, side view; e. calyx, inside view; f. opened ovary; g. placenta and ovules (all: Koch s.n., LHB 907.101-23). 
(narrowly) ovate or triangular, acuminate, c. $1.5 \mathrm{~mm}$ long. Corolla connate near the base; segments valvate; lobes (narrowly) ovate, $3.5-4$ by $1.5-2 \mathrm{~mm}$, apex acuminate; in mature buds ovoid to \pm conical, 3.5-4 mm long. Stamens c. $2 \mathrm{~mm}$ long, filaments c. $0.2 \mathrm{~mm}$ long; anthers elliptic, $0.9-1 \mathrm{~mm}$ long; connective appendages $0.9-1 \mathrm{~mm}$ long, abaxial side glandular pusticulate. Ovary ovate; placenta broadly ellipsoid; ovules 6. Style filiform, 3.4-4 mm long. Fruit globose, 7-9 mm diam. Seed globose, 3.5-5 mm diam., ribbed.

Distribution - Sumatra, Peninsular Malaysia.

Habitat \& Ecology - Usually in moist shady primary forests or damp forests at 1500-2000 m altitude. Flowering: February, March, September.

\section{Labisia sumatrensis Sunarno}

Labisia sumatrensis Sunarno (2002) 123, f. 3. - Type: De Wilde \& De Wilde-Duyfjes 19059 (holo BO; iso L), Sumatra, Aceh, upper Mamas river valley, c. $15 \mathrm{~km}$ west of Kutacane, c. $1500 \mathrm{~m}$ alt., Gn. Leuser Res., Camp central, fl. 24 June 1979.

Undershrubs, erect up to $25 \mathrm{~cm}$ tall. Stem slender, striate. Leaves spirally arranged; lamina subovate to ovate, $7-8$ by $3.5-4 \mathrm{~cm}$, subcoriaceous, base obtuse, margin crenulate, apex acute, rounded or emarginate, upper surface brownish and dull when dry, glabrous, lower surface brownish, slightly glaucous and glabrous, midrib narrowly impressed above, strongly prominent beneath, glandular punctate, secondary nerves anastomosing at 1-3 $\mathrm{mm}$ from margin; petioles terete, $3-4 \mathrm{~cm}$ long or more; base thickened. Inflorescences axillary, panicles or racemes with corymb-like fascicles, c. 4 $\mathrm{cm}$ long; peduncles c. $2 \mathrm{~cm}$ long, corymb peduncles up to $0.5 \mathrm{~cm}$ long. Bracts narrowly triangular, c. $3 \mathrm{~mm}$ long; bracteoles triangular, 1-2 mm long. Flowers pinkish white, pedicels 1-2 mm long. Calyx lobes ovate-acuminate, c. $0.5 \mathrm{~mm}$ long. Corolla connate near the base; segments valvate; lobes ovate, c. 3 by $1-1.5 \mathrm{~mm}$ long; in mature buds subovoid, c. $3 \mathrm{~mm}$ long, top acute. Stamens $1.7-1.8 \mathrm{~mm}$ long; filaments $0.4-0.6 \mathrm{~mm}$ long; anthers c. $1 \mathrm{~mm}$ long; connective appendages c. $0.3 \mathrm{~mm}$ long, backside glandular puncticulated. Ovary subglobose; placenta broadly ovoid, ovules 6 or 7. Style filiform, c. $3 \mathrm{~mm}$ long, stigma notched. Fruits unknown.

Distribution - Sumatra (East coast, Southeast Aceh, Asahan).

Habitat \& Ecology - Montane rain forests on humus-rich soils at 1200-1500 m altitude.

\section{EXCLUDED SPECIES}

Labisia malouiana L. Linden \& Rodigas (1885) 186, t. 513. - Type: Teuscher s.n. (holo: a living plant cultivated in the Botanical Garden of Brussel, Belgium), Rajah Mountains, Borneo = Ardisia malouiana (L. Linden \& Rodigas) Markgr. (1955)194.

Labisia obtusifolia Hallier f. (1901) 668. - The material cited by Hallier (Jaheri s.n., 1893, Borneo, cultivated at Bogor Botanical Garden; Teijsmann 11067) is no longer extant and no preserved specimen is available at present. The description by Hallier (1901) shows that this is an Ardisia species. 


\section{ACKNOWLEDGEMENTS}

The author would like to express his gratitude to the British Council and Flora Malesiana Foundation for financial support during his studies in the Royal Botanical Gardens, Kew and the Nationaal Herbarium Nederland, Leiden. He is grateful to R.M. Polhill, B. Verdcourt, P. Baas, the late C. Kalkman and R. Geesink for their support and encouragement of the study. He wishes to thank Mien A. Rifai and E. A. Widjaja of Herbarium Bogoriense for their advice and help. His special thanks are due to the late Dr. B.C. Stone from the Department of Botany, Academy of Natural Sciences, Philadelphia, USA for encouraging him to study the Myrsinaceae. This work was accomplished as a part of an MSc dissertation submitted to Gajah Mada University with financial support from LIPI (Lembaga Ilmu Pengetahuan Indonesia) in 1986-1989.

\section{REFERENCES}

Airy Shaw, H.K. 1939. Additions to the flora of Borneo and other Malay Islands, XIV. Bull. Misc. Inform. Kew 1939: 509-517.

Airy Shaw, H.K. 1960. Nomenclatural notes on Labisia Lindl. (Myrsinaceae). Kew Bull. 14: 320.

Airy Shaw, H.K. 1961. Further notes on Labisia pumila (Blume) Fern.-Vill. (Myrsinaceae). Kew Bull. 15: 206.

Backer, C.A. \& R.C. Bakhuizen van den Brink Jr. 1965. Flora of Java 2. Noordhoff, Groningen.

Bentham, G. \& J.D. Hooker. 1876. Genera Plantarum 2. Reeve \& Co, London.

Blume, C.L. 1823. Catalogus van eenige der merkwaardigste zoo in- als uitheemsche gewassen, te vinden in 's Lands Plantentuin te Buitenzorg, Batavia.

Blume, C.L. 1826. Bijdragen tot de flora van Nederlandsch Indië 13. Lands Drukkerij, Batavia.

Brown, N.E. 1886. Labisia alata N.E. Brown. Ill. Hort. 33: 123, t. 605.

Clarke, C.B. 1882. Myrsineae. In: J.D. Hooker, Fl. Brit. India 3: 507-534. Reeve \& Co, Brook, nr. Ashford.

De Candolle, A. 1834. Review of the Natural order Myrsineae. Trans. Linn. Soc. London 17: 95137.

Fernández-Villar, C. 1880. Labisia. In: A. Naves \& C. Fernández-Villar, Novissima Appendix.

Fletcher, H.R. 1950. Keys to the Siamese species of Myrsinaceae. Notes Roy. Bot. Gard. Edinburgh 20: $112-120$.

Hallier, H. 1887. Die indonesischen Clematideen des Herbariums zu Buitenzorg. Ann. Jard. Bot. Buitenzorg 14: 248-276.

Hallier, H. 1901. Neue und bemerkenswerte Pflanzen aus dem malaiisch-papuanischen Inselmeer. III. Teil. Bull. Herb. Boissier sér. 2, 1: 667-676.

Hallier, H. 1915. Neue und bemerkenswerte Pflanzen aus der malaiisch-papuanischen Inselwelt. Meded. Rijks-Herb. 26: 1-8.

Hochreutiner, B.P. G. 1925. Plantae Hochreutineranae. Étude systematique et Biologique des collections faites par l'auteur au cours de son voyage aux Indes Neerlandaises et autour du monde pendant les annales 1903 à 1905. Fascicule II. Candollea 2: 317-513.

Jackson, B.D. 1895. Index Kewensis 2. Clarendon Press, Oxford.

Kanehira, R. \& S. Hatusima. 1943. The Kanehira-Hatusima collection of New Guinea Plants xxi. Myrsinaceae. Bot. Mag. (Tokyo) 57: 215-236.

King, G. \& J.S. Gamble. 1905. Materials for a flora of the Malay Peninsula, no. 17. J. Asiat. Soc. Bengal 74, 2: 93-273.

Linden, L. \& E. Rodigas. 1885. Labisia malouiana L.L. Linden et Em. Rod. Ill. Hort. 32: 186, t. 513.

Linden, L. \& E. Rodigas. 1892. Labisia smaragdina Linden \& Rodigas. Ill. Hort. 39: 91, 92, t. 160; 140, t. 109.

Lindley, J. 1845. Miscellaneous notices. Bot. Reg. n.s. 31: 48, 49.

Markgraf, F. 1955. Eine bluntblättrige Myrsinacee der Botanischen Garten. Gartenbauwissenschaft 20: $192-194$.

Masamune, G. 1942. Enumeration Phanerogamae Borneense. 
Mez, C. 1902. Myrsinaceae. In: A. Engler, Das Pflanzenreich IV, 236: 1-437.

Merrill, E.D. 1907. The flora of Mount Halcon, Mindoro. Philipp. J. Sci., Bot. 2: 251-309.

Merrill, E.D. 1923. An enumeration of Philippine flowering plants 3. Bureau of Printing, Manila.

Miquel, F. A.W. 1856. Flora Indiae Batavae 2. Van der Post, Amsterdam.

Pax, F. 1889. Myrsinaceae. In: A. Engler \& K. Prantl, Die natürlichen Pflanzenfamilien 4, 1: 81-97. Engelmann, Leipzig.

Pitard, J. 1930. Myrsinaceae. In: H. Lecomte, Fl. Indo-Chine 3: 765- 877.

Reinwardt, C.G.C. 1826. Nova plantarum indicarum genera. Sylloge Plantarum novarum. Regensburg 2: 1-15.

Ridley, H.N. 1908. New or rare Malayan plants. Series IV. J. Straits Branch Roy. Asiat. Soc. 50: $111-152$.

Ridley, H.N. 1923a. The Flora of the Malay Peninsula, 2. - Gamopetalae. Reeve \& Co, London.

Ridley, H.N. 1923b. A botanical excursion to Northern Sumatra. J. Malayan Branch Roy. Asiat. Soc. 1: 46-113.

Scheffer, R.H.C.C. 1867. Commentatio de Myrcinaceis archipelagi indici. Bergman, Weesp.

Stone, B.C. 1981. The summit flora of Gunung Ulu Kali (Pahang, Malaysia). Fed. Mus. J. 26: 1-157.

Stone, B.C. 1988. Notes on the genus Labisia Lindl. (Myrsinaceae). Malayan Nat. J. 42: 43-51.

Sunarno, B. 2002. New species of Labisia (Myrsinaceae) from Sumatra. Reinwardtia 12: 121-124.

Valeton, Th. 1907. Plantae Papuanae. Bull. Dép. Agric. Indes Néerl. 10: 1-70.

Van Steenis, C.G.G.J. 1948. Proposal VI. Conservation of Labisia Lindl. against Angiopetalum Reinw. Fl. Males. Bull. 1: 109, 110.

\section{IDENTIFICATION LIST}

1. L. acuta

2. L. longistyla

3. L. paucifolia

4. L. posthumusiana

5. L. pumila var. alata

6. L. pumila var. discoplacenta

7. L. pumila var. gladiata
8. L. pumila var. lanceolata
9. L. pumila var. malintangensis
10. L. pumila var. neriifolia
11. L. pumila var. pumila
12. L. pumila var. sessilifolia
13. L. steenisiana
14. L. sumatrensis

Aet 508: 8 - Afriastini 26: 8; 332: 1; 688: 8; 1288: 6 - Ajoeb 89: 8 - Amdjah 1046: 1 - Ashton 73: $11 ; 246: 8 ; 301: 11 ; 448: 8$ - Argent et al. 108240: 8 .

Backer 6384: 11; 7088: 5; 7177: 11; 10133: 8; 10876: 8; 10983: 8; 14236: 8; 14499: 8; 14602: 11; 15099: 8; 22663: 11; 23063: 11; 25747: 11; 25925: 11 - Bakhuizen van den Brink 846: 11; 1384: 5; 1462: 8; 2743: 11; 4340: 8; 4364: 5; 4759: 5 - Barber 79: 5 - Beaman 7016: 8; 7777: 8; 8351: 8 - Boden Kloss 64: 8; 14746: 11 - Brooke 8027: 5; 8243: 8; 8499: 7; 8765: 8; 10240: 8; 10425: 8; 10763: 10 — Bünnemeijer 379: 5; 1089: 11; 1790: 9; 2104: 1; 3328: 5; 3472: 5; 3632: 9; 4282: 5; 5910: 5 .

Castle-Smith 10: 5; 50: 2 - Charoemphol et al. 3485: 5 - Chew Wee-Lek 809: 2; 1425: 8 - J. \& M.S. Clemens 27055: 5; 30789: 11; 40554: 8 - Corner SF 30232: 5 - Curtis 11: 8; 3750: 2.

De Vogel 1167: 5; 1295: 11; 4085: 12 — De Wilde \& De Wilde-Duyfjes 12323: 5; 12363: 5; 12765 : 5; 12855: 8; 14881: 5; 18310: 14; 19059: 14 - Derry 11: 8 - Dransfield 1007: 5.

Elbert 3151: 10; 3757: 10 - Endert 1611: 8; 1911: 8; 2763: 8; 2978: 10; 2984: 8 - Eyma 2421: $11 ; 2753: 11$.

Forbes 114: 8; 238: 11; 474: 9; 1990: 11 — Franken \& Roos 18: 1; 351: 4 - FRI 4188 (Ng): 8; $5334(\mathrm{Ng}): 5 ; 5458$ (Ng): 8; 5671: 11; 12691: 8; 15537 (Whitmore): 2; 19393 (Kochummen): 2; 26211 (Kochummen): 8.

Geesink \& Santisuk 4969: 5; 5161: 11; 5398: 5 - George S 42818: 8 - Griffith 3559: 5.

Hallier 492: 10; 2428: 10; 2893: 8; 3423: 8 - Hassan \& Kadim SFN 19: 8 - Haviland 551: 8; 643: 8; 1738: 8; 2201: 8 - Holttum 10620: 8 - Ding Hou 193: 8; 674: 5 - Hsuan Keng et al. 81: 8. 
Iboet 45: 3; 113: 3 - Iwatsuki et al. S-1080: 11.

Jacobs 8411: 5 - Jaheri 160: 10.

Kadim \& Noor SIBG 225: 8 - Kalong 22443: 2 - Kanehira \& Hatusima 12574: 12; 12707: 12 - Kerr 6830: 5; 8568: 5; 8602: 5; 10868: 5; 12563: 5; 14383: 5; 14508: 5; 15732: 5; 15769: 11; 18515: 11; 18984: 11; 24553: 8 - King 2882: 5; 8325: 5 - Kjellberg 1759: 8; 2893: 8 - Koorders 1220: 11; 6059: 11; 22341: 5; 22342: 1; 33411: 11; 39434: 11; 39437: 11; 39499: 11; 39514: 8; 39519: 5; 40838: 8 - Kostermans 1281: 8; 6302: 5; 12776: 8; 12936: 8; 12946: 8; 13060: 8 - Kostermans \& Anta 811: 8.

Leighton 671: 8 - Lobb 61: 8; 313: 5 - Lörzing 5353: 5; 5752: 5; 5797: 8; 5805: 11; 5910: 11; 6592: 9; 7810: 11; 10025: 8; 14888: 8; 17336: 5; 41029: 5.

Machille 4750: 8 - Maingay 1013: 5 - Maxwell 78-68: 8; 80-116: 10; 82-74: 8 - Meijer 893: 8; 6763: 8; 6894: 5; 8695: 5 - Mogea 3653: 8 - Motley 79: 5; 1250: 8 - Murata et al. B-1439: 8; B-4885: 9 .

Native collector 417: 8 - Nedi 617: 8 - Noerkas 490: 10 - Noraini 5946: 3; 6852:3 - Nooteboom 4169: 8 - Nooteboom \& Aban 681: 11; 1183: 11; 1509: 11 - Moh. Nur 7395: 5 - Nyondham 849: 8.

Okada \& Mori 1214: 2.

Phusomsaeng 233: 5 - Polak 620: 1 - Posthumus 577: 8; 777: 4 - Pringgoatmodjo 139: 8; 196: 8; 495: 5 - Purseglove 4207: 2; 4394: 11; 4935: 5 - Put 1760: 11.

Rachmat 471: 10; 861: 10 - Rahmat si Boeea 6229: 8; 10202: 14; 10313: 8; 10633: 14; 10660: 14; 10910: 8; 11181: 3 - Razali 1864: 3 - Richards 1656: 8; 1847: 8; 2112: 8; $2113: 9$ - Ridley 1804: 8; 2199: 8; 2199a: 8; 13010: 1; 15642: 2 - Ridsdale \& Galor NGF 33449: 12 - Riswan 361: 5 - Robinson \& Boden Kloss 6125: 8.

S series 234 (Kiah): 8; 14755 (A. Muas): 8; 19548 (P. Chai): 8; 19687 (Anderson): 8; 21662 (Mohidin): 8; 33319 (P. Chai): 8; 33977 (P. Chai): 8; 36862 (Martin \& Ismawi): 8; 41651 (I. Paie): 8; 43744 (Othman et al.): 8 - Said 1174: 2 - SAN 26862 (Bakar): 8; 49538 (Sadau): 8; 79079 (Sundaling): 8; 83051 (Cockburn): 11; 83431 (Madani): 8; 87702 (Tarodop): 11; 88352 (Fedilis \& Sumbing): 8; 90996 (Madani \& Saigol): 8; 93299 (Rahim et al.): 8 - Schmutz 5818: 9 - SF 434 (Allen \& Kadim): 5; 456 (Shah): 8; 489 (Allen \& Kadim): 8; 728 (Shah \& Nur): 8; 903 (Shah \& Nur): 11; 1761 (Shah \& Nur): 5; 1927: 8; 2081 (Shah et al.): 8; 2687 (Shah \& Shukor): 5; 4298 (Purseglove): 11; 31795: 5; 32172: 8; 34346: 8; 35104 (Kiah): 5; 36643 (Ngadiman): 8 - Shea 23139: 8; 23141: 10; 23282: 8; 23925: 11; 72002: 8 - Siew Wei Hoe 171: 3 - Smith BE 89: 14 - Soepadmo 59: 8 - Sow CF 47186: 2 - Stevens et al. 249: 1; 362: 10 - Streimann LAE 51744: 12 - Sunarno 40: 1; 184: 8; 252: 1; 637: 12 - Symington 32227: 2 - Synge 50: 7; 410: 8; 1081: 11 .

Toxopeus 212: 12; 222: 10 - Tukirin 659: 1.

Van Balgooy 2115: 8; 4069: 10 - Van Balgooy \& Wiriadinata 2899: 8 - Van Beusekom \& Phengkhlai 6899: 11 - Van Borssum Waalkes 1543: 5; 2344: 5 - Van Leeuwen-Van Reijn 2869: 8 - Van Niel 4115: 8 - Van Royen \& Sleumer 7597: 12 - Van Steenis 1415: 5; 2761: 8; 3571: 11; 3746: 5; 6310: 13; 8702: 13; 9498: 5; 9546: 13; 10087: 5 - Vermeulen 783: 5 - Vreeken-Buijs 6: 4.

Walker 165: 8 - Wenzel 666: 11 - Winkler 2528: 8 - Wiriadinata \& Maskuri 698: 8 - Wray 2482: 5 - Wyatt-Smith 94592: 2.

Yates 2356: 11 - Yutieng et al. PNH 117395: 5. 


\section{INDEX}

Accepted names appear in roman type, synonyms in italics, and new names are printed in bold. The number after each name is the number of the species in the treatment; excl. $=$ excluded species.

Allopetalum punctatum Reinw. ex Scheff. $5,5 \mathrm{~b}$

Angiopetalum Reinw. [p. 579] punctatum Reinw. 5, 5b

Ardisia bantamica Zipp. ex Scheff. 5 malouiana (L. Linden \& Rodigas) Markgr. excl.

pumila Blume 5

$\alpha$ genuiana Scheff. 5a

$\beta$ lanceolata Scheff. 5, 5e

$\gamma$ alata Scheff. 5, 5b

spicata Wall. ex DC. 5

Labisia Lindl. [p. 580]

acuta Ridl. 1

alata (Scheff.) N.E. Br. 5, 5b

longistyla King \& Gamble 2

malouiana L. Linden \& Rodigas excl.

neriifolia Hallier f. 5, 5g

obtusifolia Hallier f. excl.

ovalifolia Ridl. 5, 5b

paucifolia King \& Gamble 3

posthumusiana Sunarno 4

pothoina Lindl. 5

var. alata (Scheff.) King \& Gamble 5, 5b

var. lanceolata (Scheff.) King \& Gamble $5,5 \mathrm{e}$

pumila (Blume) Benth. \& Hook.f. ex Jacks. 5

pumila (Blume) Fern.-Vill. 5

$\alpha$ genuiana Mez 5 a

$\beta$ alata auct. $5 \mathrm{~h}$ (pumila (Blume) Fern.-Vill.)

var. alata (Scheff.) Mez 5, 5b

var. discoplacenta Sunarno $5 \mathrm{c}$

var. gladiata (Airy Shaw) Masam. 5d

var. lanceolata (Scheff.) Mez 5, 5e

forma sessilifolia Valeton 5, 5h

var. malintangensis Sunarno $5 \mathrm{f}$

var. neriifolia (Hallier f.) Sunarno $5 \mathrm{~g}$

var. pumila $5 \mathrm{a}$

var. sessilifolia (Valeton) Sunarno $5 \mathrm{~h}$

forma genuiana Mez ex Airy Shaw 5a

forma gladiata Airy Shaw 5d

forma lanceolata (Scheff.) Mez ex

Airy Shaw 5, 5e

pumila (Blume) Mez ex Airy Shaw 5

pumila (Reinw.) Airy Shaw

var. gladiata (Airy Shaw) Masam. 5

forma gladiata Airy Shaw 5

punctata Reinw. 5b

forma punctata Airy Shaw $5 \mathrm{~b}$

punctata (Reinw.) Airy Shaw 5

forma gladiata (Airy Shaw) Airy Shaw $5,5 \mathrm{~d}$

forma lanceolata (Scheff.) Airy Shaw

$5,5 \mathrm{e}$

punctata (Reinw.) Lindl.

forma pumila (Blume) Airy Shaw 5a

serrulata Hallier f. 5, 5b

sessilifolia (Valeton) Hallier f. 5

smaragdina L. Linden \& Rodigas 5

steenisiana Sunarno 6

sumatrensis Sunarno 7 